\title{
Analytical Investigation of the Behavior and Distribution of Dust Particles Trapped in Horseshoe Orbit
}

\author{
By Norizumi MotooKA ${ }^{1)}$,Osamu Mori ${ }^{2)}$ \\ and Jun'ichiro KAWAGUCHI ${ }^{2}$ \\ ${ }^{1)}$ Department of Aeronautics and Astronautics, The University of Tokyo, Tokyo, Japan \\ ${ }^{2)}$ Japan Aerospace Exploration Agency, Sagamihara, Japan
}

(Received June 27th, 2011)

\begin{abstract}
This paper analyzes the behavior of an interplanetary dust particle on a horseshoe orbit by solving equations of the circular restricted three-body problem (CR3BP) analytically to predict the distribution of dust particles around the Earth's orbit, because in previous research numerical simulation showed that dust particles trapped on a horseshoe orbit contributes a number density of dust particles around the Earth's orbit. An analytical solution is obtained by coordinate transformation and linearization of external forces on the assumption that a horseshoe orbit is an oscillation centered at Lagrange point L3. This paper describes properties of the horseshoe solution and also shows that the analytical solution of the CR3BP is consistent with that of numerical integration.
\end{abstract}

Key Words: Interplanetary Dust Particles, Horseshoe Orbit, Solar Radiation Pressure, Poynting Robertson Drag

\section{Nomenclature}

$\mu \quad:$ gravitational constant

$\boldsymbol{r} \quad$ : position of a dust in the rotating frame

$\boldsymbol{V} \quad$ : velocity of a dust in the rotating frame

$v \quad:$ velocity of a dust in the inertial frame

$n \quad:$ mean motion of the Earth

$c \quad:$ speed of light

$\beta \quad$ : ratio of solar radiation pressure to solar gravity

$r_{0} \quad: \quad$ the average distance between the Sun and Earth

$\omega \quad$ : angular velocity of coordinate system

$f \quad$ : force acting on a dust

Subscripts

$S \quad$ : related to the Sun

$E \quad$ : related to the Earth

$S R P \quad$ : Solar Radiation Pressure

$P R \quad$ : Poynting-Robertson drag

\section{Introduction}

Interplanetary dust particles are observed from an astronomical satellite as thermal flux of infrared ray. For infrared space observatory, removal of the infrared radiation from dust particles brings higher quality of scientific observation. Therefore, to produce the precise model on the distribution around Earth's orbit has been required. In our previous study ${ }^{1)}$, a distribution was predicted and calculated numerically on the assumption that dust particles which are trapped in a horseshoe orbit by the Earth's gravity contribute the distribution. And our study also confirmed the possibility of a dust particle trapped in a horseshoe orbit by numerical simulations. A horseshoe orbit is a numerical solution of the circular restricted three-body problem (CR3BP) which encompasses Lagrange point L3, L4 and L5. But not only numerical solution but also analytical one are important, because the analytical solution can clearly show the cause-and-effect relationship between external forces acting on a dust and its distribution. Therefore, this paper investigates the behavior of a dust particle on a horseshoe orbit by solving the CR3BP which incorporates solar radiation pressure (SRP) and Poynting-Robertson (P-R) drag as major perturbation sources.

\section{Forces on Dust Particles}

This paper focuses on micrometer-size dust particles which lie within a plane of the ecliptic. These origins are, by the recent observational evidence, asteroids and comets. Dust particles which leave from their origins lose orbital energy by P-R drag and spiral in towards the Sun on timescales $\sim 10^{4}$ year. A dust is subject to non-gravitational forces such as SRP, solar wing, attracting force between dust particles, and mutual interaction (Lorentz force) between an electrically charged dust and interplanetary magnetic field. The principal perturbation force of them is SRP. A component of SRP acts as a drag force known as P-R drag. This research investigated the motion of a dust by analyzing CR3BP which incorporates $\mathrm{SRP}$ and P-R drag as perturbation forces.

\subsection{Motion equation of a dust}

The equation of motion in CR3BP in Cartesian coordinates $(X, Y, Z)$ is the following.

$$
\ddot{r}+2 \omega \times \dot{r}+\omega \times(\omega \times \dot{r})=f_{S}+f_{E}+f_{S R P}+f_{P R}
$$


Where,

$$
\begin{aligned}
& \boldsymbol{r}=\left(\begin{array}{l}
X \\
Y \\
X
\end{array}\right), \boldsymbol{\omega}=\left(\begin{array}{l}
0 \\
0 \\
n
\end{array}\right), \\
& \boldsymbol{f}_{S}=-\frac{\mu_{S}}{r_{S}^{2}} \frac{\boldsymbol{r}_{S}}{r_{S}}, \boldsymbol{f}_{E}=-\frac{\mu_{E}}{r_{E}^{2}} \frac{\boldsymbol{r}_{E}}{r_{E}}, \boldsymbol{f}_{S R P}=\beta \frac{\mu_{S}}{r_{S}^{2}} \frac{\boldsymbol{r}_{S}}{r_{S}}
\end{aligned}
$$

$r_{S}$ and $r_{E}$ are the magnitude of the vector $\boldsymbol{r}_{S}$ and $\boldsymbol{r}_{E}$ respectively. If a dust is sphere, SRP reduces the gravity of Sun.

$$
\boldsymbol{f}_{S}+\boldsymbol{f}_{E}+\boldsymbol{f}_{S R P}=-(1-\beta) \frac{\mu_{S}}{r_{S}^{2}} \frac{\boldsymbol{r}_{S}}{r_{S}}-\frac{\mu_{E}}{r_{E}^{2}} \frac{\boldsymbol{r}_{E}}{r_{E}}
$$

\subsection{Poynting-Robertson $\mathrm{drag}^{3,4)}$}

P-R drag is a process by which SRP causes a dust particle in the solar system to slowly spiral inward. It was named after its discoverers, John Henry Poynting and Howard Percy Robertson. The drag is essentially a component of SRP tangential to the direction of the dust's motion. From perturbed equation, dust's eccentricity and semimajor axis decay due to P-R drag with time. The drag force makes dust particles spiral into the Sun where these particles evaporate. The force of P-R drag on a dust is written as.

$$
\boldsymbol{f}_{\boldsymbol{P R}}=-\beta \frac{\mu_{S}}{r_{S}^{2}}\left(\frac{\left(\boldsymbol{v} \cdot \boldsymbol{r}_{S}\right)}{c} \frac{\boldsymbol{r}_{S}}{r_{S}^{2}}+\frac{\boldsymbol{v}}{c}\right)
$$

\section{Horseshoe Orbit}

A horseshoe orbit is a numerical solution of the CR3BP and encompasses Lagrange point L3, L4, and L5 in Sun-Earth rotating frame as Fig. 2. shows. A dust trapped in a horseshoe orbit turns ahead of and behind of the Earth by its gravity in the rotating frame. Numerical integrations revealed that a velocity of a dust on a horseshoe orbit is not constant and becomes a minimum at turned edges of the orbit. This change of velocity on the orbit produces a density distribution of dust particles around the Earth's orbit.

\section{Modeling of a Horseshoe Orbit}

It is important to solve the CR3BP incorporating perturbation forces analytically to understand a dust's behavior on a horseshoe orbit. A horseshoe solution was obtained by linearizing the external forces on the assumptions that a horseshoe orbit is an oscillation about Lagrange point L3 in Sun-Earth rotating polar coordinates in Fig. 3. This modeling revealed that a horseshoe solution consisted of two oscillatory one and represented properties of a horseshoe orbit predicted by the numerical integration.

\subsection{A horseshoe orbit subjected to only gravitational} forces

\subsubsection{Linearization of motion equations of CR3BP}

The motion equation of CR3BP without perturbation equation in polar coordinate system $(r, \theta, z)$ is expressive as,

$$
\ddot{\boldsymbol{r}}+2 \boldsymbol{\omega} \times \dot{\boldsymbol{r}}+\boldsymbol{\omega} \times(\boldsymbol{\omega} \times \boldsymbol{r})+\dot{\boldsymbol{\omega}} \times \boldsymbol{r}=\boldsymbol{f}_{S}+\boldsymbol{f}_{E}
$$

where,

$$
\begin{gathered}
\boldsymbol{r}=\left(\begin{array}{c}
r \\
\theta \\
z
\end{array}\right), \boldsymbol{\omega}=\left(\begin{array}{c}
0 \\
0 \\
n+\omega_{p}
\end{array}\right) \\
\boldsymbol{f}_{S}=\left(\begin{array}{c}
f_{S_{-} r}(r, \theta) \\
f_{S_{-} \theta}(r, \theta) \\
0
\end{array}\right), \boldsymbol{f}_{E}=\left(\begin{array}{c}
f_{E_{-} r}(r, \theta) \\
f_{E_{-} \theta}(r, \theta) \\
0
\end{array}\right)
\end{gathered}
$$

$\omega_{p}$ is angular velocity of the polar coordinate system $(r, \theta, z)$ relative to Cartesian coordinates $(X, Y, Z)$.

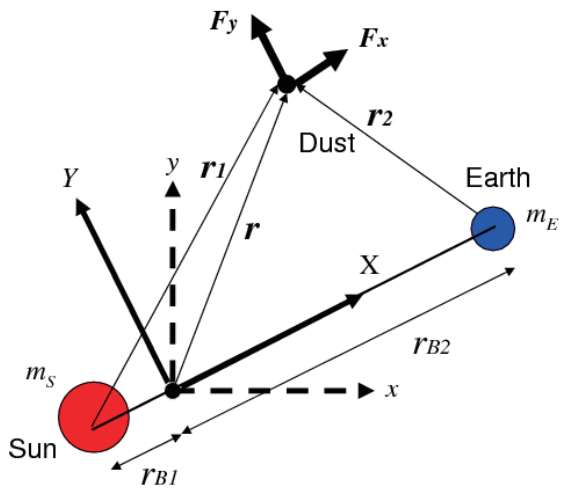

Fig. 1. Geometry for the Restricted Three-body Problem: Notice the $\mathrm{X}$-axis is in the direction of the Earth, and the $\mathrm{Y}$-axis lies in a plane of the ecliptic. This synodic system is rotating with respect to the fixed barycentric frame $(\mathrm{x}, \mathrm{y}, \mathrm{z})$.

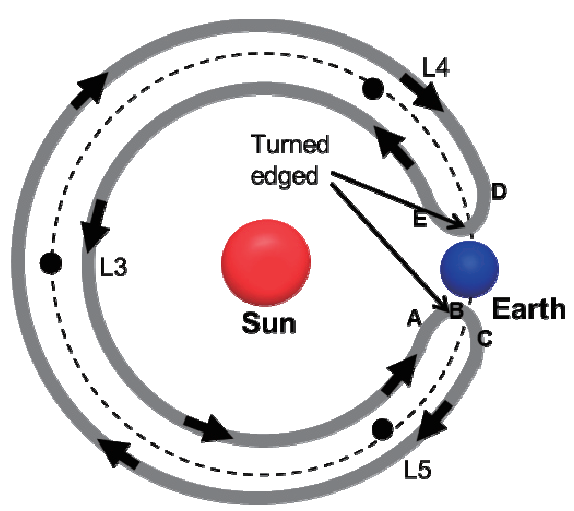

Fig. 2. Horseshoe orbit in Sun-Earth rotating frame.

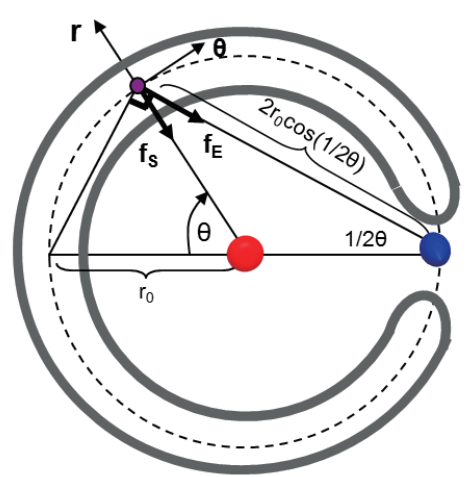

Fig. 3. Geometrical definition of variables in linearization of CR3BP. 
To linearize gravities of Earth and Sun, we assumed the following preconditions

1. Neglect the change of angular velocity derived from coordinate conversion

$$
\omega_{p}=0
$$

2. The gravity center of the Sun is equal to that of the Sun and the Earth

$$
\boldsymbol{r}=\boldsymbol{r}_{S}
$$

Using the geometric relationship in Fig. 3, the equation (4.1) becomes,

$$
\left[\begin{array}{c}
\ddot{r} \\
r_{0} \ddot{\theta}
\end{array}\right]+\left[\begin{array}{c}
-2 n r_{0} \dot{\theta} \\
2 n \dot{r}
\end{array}\right]-\left[\begin{array}{c}
n^{2} r \\
0
\end{array}\right]=\left[\begin{array}{c}
-\frac{\mu_{S}}{r^{2}} \\
0
\end{array}\right]+\left[\begin{array}{c}
-\frac{\mu_{E}}{\left(2 r_{0}\right)^{2} \cos \frac{1}{2} \theta} \\
\frac{\mu_{E}}{\left(2 r_{0} \cos \frac{1}{2} \theta\right)^{2}} \sin \frac{1}{2} \theta
\end{array}\right]
$$

Linearized gravities of the Sun and the Earth are obtained by Taylor expanding $\boldsymbol{f}_{S}$ and $\boldsymbol{f}_{E}$ about $r=r_{0}$ and $\theta=\theta_{0}=0$, respectively, on the assumption that a horseshoe orbit is the oscillation centered about L3,

$$
r=r_{0}+\delta r, \theta=\delta \theta \quad \delta r \square 1, \delta \theta \square 1
$$

$r_{0}$ : distance between the Sun and the Earth

Linearized equations about L3 are

$$
\begin{aligned}
& \frac{\delta \ddot{r}}{n^{2} r_{0}}-2 \frac{\delta \dot{\theta}}{n}-\left(1+\frac{\delta r}{r_{0}}\right)=-\frac{\mu_{S}}{n^{2} r_{0}{ }^{3}}\left(1-2 \frac{\delta r}{r_{0}}\right)-\frac{\mu_{E}}{4 n^{2} r_{0}{ }^{3}} \\
& \frac{\delta \ddot{\theta}}{n^{2}}+2 \frac{\delta \dot{r}}{n}=\frac{\mu_{E}}{8 n^{2} r_{0}{ }^{3}} \delta \theta
\end{aligned}
$$

We can normalize Eq. (4.8) by using the following relationship,

$$
\begin{aligned}
& \delta r=r_{0} \delta r^{\prime}, \delta \dot{r}=n^{2} r_{0} \delta \dot{r}^{\prime}, \delta \ddot{r}=n^{2} r_{0} \delta \ddot{r}^{\prime} \\
& \delta \theta=\delta \theta^{\prime}, \delta \dot{\theta}=n \delta \dot{\theta}^{\prime}, \delta \ddot{\theta}=n^{2} \delta \ddot{\theta}^{\prime} \\
& \mu_{S}=\mu_{2} n^{2} r_{0}^{3}, \mu_{E}=\mu_{1} n^{2} r_{0}^{3}, c=c^{\prime} n r_{0}
\end{aligned}
$$

Substituting Eq. (4.9) into Eq. (4.8) and using the approximation $\mu_{2} \approx 1$ gives Eq.(4.10)

$$
\begin{aligned}
& \delta \ddot{r}^{\prime}-2 \delta \dot{\theta}^{\prime}-3\left(\delta r^{\prime}-\frac{\mu_{1}}{12}\right)=0 \\
& \delta \ddot{\theta}^{\prime}+2 \delta \dot{r}^{\prime}=\frac{\mu_{1}}{8} \delta \theta
\end{aligned}
$$

that is,

$$
\begin{aligned}
& \ddot{r}-2 \dot{\theta}-3 r=0 \\
& \ddot{\theta}+2 \dot{r}-\frac{\mu_{1}}{8} \theta=0
\end{aligned}
$$

where the transformation of variables

$$
\delta r^{\prime}-\frac{\mu_{1}}{12} \rightarrow r, \delta \theta \rightarrow \theta
$$

\subsubsection{Solution of linearized motion equation}

Solutions for differential equations Eq. (4.11) are the following.

$$
\begin{gathered}
r=A_{l} \cos \left(\omega_{l} t+\gamma_{l}\right)+A_{s} \cos \left(\omega_{s} t+\gamma_{s}\right) \\
\theta=B_{l} \sin \left(\omega_{l} t+\gamma_{l}\right)+B_{s} \sin \left(\omega_{s} t+\gamma_{s}\right) \\
\left\{\begin{array} { c } 
{ \omega _ { s } = 0 . 9 9 9 9 9 9 } \\
{ \omega _ { l } = 1 . 0 6 1 2 8 \cdot 1 0 ^ { - 3 } }
\end{array} \rightarrow \left\{\begin{array}{l}
\mathrm{T}_{s}=1.00[\text { year }] \\
\mathrm{T}_{l}=942[\text { year }]
\end{array}\right.\right.
\end{gathered}
$$

The analytical solution consists of long and short periodic one. The long period solution determines the shape of a horseshoe orbit and the short period solution derives from the difference of eccentricity between a dust and the Earth. Fig. 4. shows a horseshoe orbit of a dust which has a small eccentricity $(\mathrm{e}=0.05)$.

\subsubsection{Distribution of dust particles on a horseshoe orbit}

In the case of $e=0$, velocity of a dust on a horseshoe orbit is given by formula

$$
\begin{gathered}
v^{2}=\dot{r}^{2}+(r \dot{\theta})^{2} \\
=A_{l} \omega_{l}\left(A_{l} \omega_{l}-B_{l}\right) \sin ^{2} \Omega_{l}+A_{l} B_{l} \omega_{l} \\
\Omega_{l}=\omega_{l} t+\gamma_{l}
\end{gathered}
$$

The velocity of a dust becomes minimum at $\Omega_{l}=\pi / 2+2 n \pi, 3 \pi / 2+2 n \pi$, which means that the velocity of a dust on a horseshoe orbit becomes minimum at turned edges of the orbit. Fig. 5 shows a horseshoe orbit calculated numerically and Fig. 6 shows the velocity distribution of a dust particle on the orbit in Fig. 5. If dust particles are trapped in a horseshoe orbit at the same frequency, the number density of a dust becomes maximum at turned edges of the horseshoe orbit (phase angle $=0,360$ deg in Fig. 5).

\subsubsection{Time cycle of a horseshoe orbit}

In the analytical solution we obtained, time cycle of a horseshoe orbit was determined uniquely by the coefficients of Eq. (4.11) and doesn't depend on the amplitude of the oscillation, that is, the width of a horseshoe orbit. In numerical simulation, however, the time cycle varied with the width of a horseshoe orbit. This property was lost in the process of linearization of the Earth's gravity. So, to reflect this property in the analytical solution, we modified the Eq. (4.11) using the variational method.

The modified equation incorporating the third order approximation of $f_{E \theta} \theta$ is

$$
\begin{aligned}
& \ddot{r}-2 \dot{\theta}-3 r=0 \\
& \ddot{\theta}+2 \dot{r}-\frac{\mu_{1}}{8}\left(\theta+\frac{5}{24} \theta^{3}\right)=0
\end{aligned}
$$

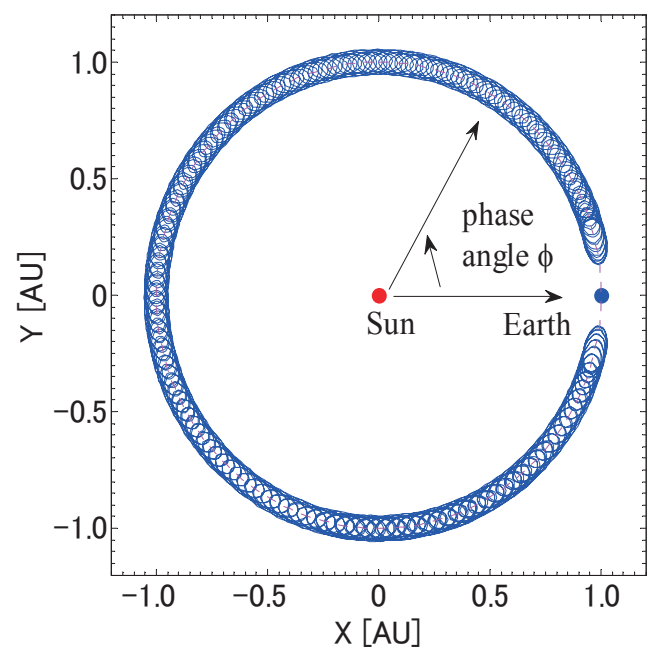

Fig. 4. A horseshoe orbit obtained by numerical simulation: A dust particle moves along the horseshoe orbit by drawing small circles. The orbit has small eccentricity $(\mathrm{e}=0.05)$ in the inertial frame. 
where the fourth term in the second equation of Eq. (4.17) derives from the third order approximation of $f_{E_{-} \theta}$, Taylor expanding $f_{E_{-} \theta}$ is the following

$$
f_{E_{-} \theta}\left(r_{0}, \delta \theta\right)=\frac{\mu_{E}}{8 r_{0}^{2}}\left\{\delta \theta+\frac{5}{24} \delta \theta^{3}+O(\delta \theta)^{4}\right\}
$$

The long periodic solution of Eq. (4.17) can be expressed as Eq. (4.18).

$$
\begin{aligned}
& r=A_{l} \cos \left(\omega_{l} t+\gamma_{l}\right) \\
& \theta=B_{l} \sin \left(\omega_{l} t+\gamma_{l}\right)
\end{aligned}
$$

where the angular velocity of a dust is a function of the width of a horseshoe orbit

$$
\omega_{l}=\omega_{l}\left(A_{l}\right)
$$

Eq. (4.17) can be solved analytically using a variational method. Stationary value is

$$
\frac{\partial}{\partial A}\left\{\int_{t}^{t+T} L(t, A) d t\right\}=0
$$

where the Lagragian

$$
\begin{aligned}
L=\frac{1}{2}\left\{(\dot{r}-\theta)^{2}+(\dot{\theta}+r)^{2}\right\} & \\
& +r^{2}+\left(\frac{\mu}{16}-\frac{1}{2}\right) \theta^{2}+\frac{\mu}{8} \frac{5}{24} \frac{\theta^{4}}{4}
\end{aligned}
$$

was used.

Fig. 7 shows the result of analysis using variational method. The analytical solution was improved by considering third order approximation of the Earth's gravity.

\subsection{A horseshoe orbit subjected to SRP}

By substituting the precondition Eq. (4.5) into Eq. (2.2), we can obtain.

$$
f_{S R P}=\frac{\beta \mu_{S}}{r^{2}} \frac{r}{r}
$$

Linearized equation including SRP is

$$
\begin{aligned}
& \ddot{r}-2 \dot{\theta}-(3-2 \beta) r=0 \\
& \ddot{\theta}+2 \dot{r}-\frac{\mu_{1}}{8} \theta=0
\end{aligned}
$$

where the transformation of variables

$$
\begin{gathered}
\left(\delta r^{\prime}+\Delta a\right) \rightarrow r, \delta \theta \rightarrow \theta \\
\Delta a=\frac{\beta-1 / 4 \mu_{1}}{3-2 \beta} \approx 0.036 A U(\beta=0.1)
\end{gathered}
$$

In the case of $\beta=0.1$, time cycle is

$$
\left\{\begin{array} { c } 
{ \omega _ { s } = 1 . 0 9 5 } \\
{ \omega _ { l } = 9 . 3 4 0 \cdot 1 0 ^ { - 4 } }
\end{array} \rightarrow \left\{\begin{array}{c}
\mathrm{T}_{s}=0.913 \text { year } \\
\mathrm{T}_{l}=1068 \text { year }
\end{array}\right.\right.
$$

From analytical solutions, SRP translates center of oscillation about radius direction toward the Sun by $\Delta a$. And time cycle of short period solution decreases and that of long one increases. This property was confirmed by numerical simulation in Fig. 8.

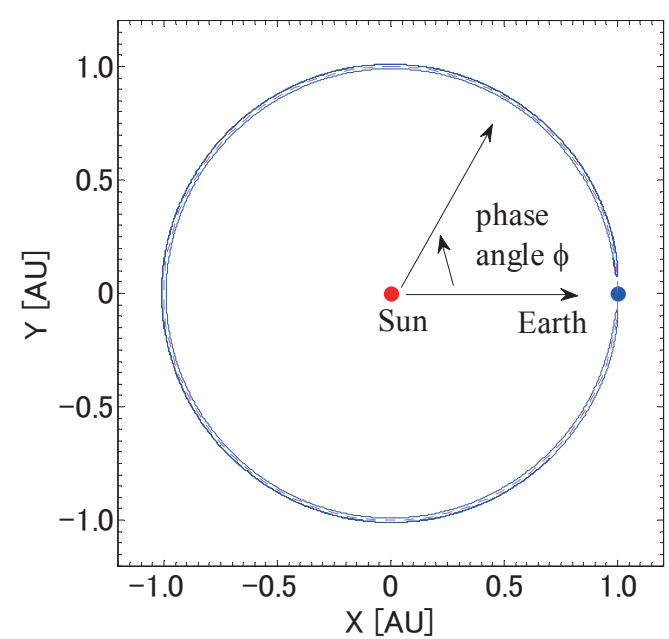

Fig. 5. Horseshoe orbit obtained numerically ( $\beta=0, \mathrm{~T}=150 \mathrm{year}$ ).

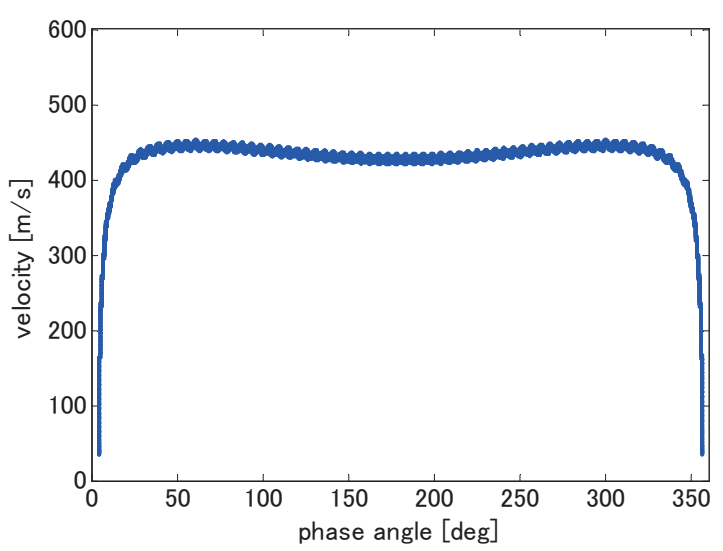

Fig. 6. Velocity distribution of a dust trapped in a horseshoe orbit in Fig. 5.

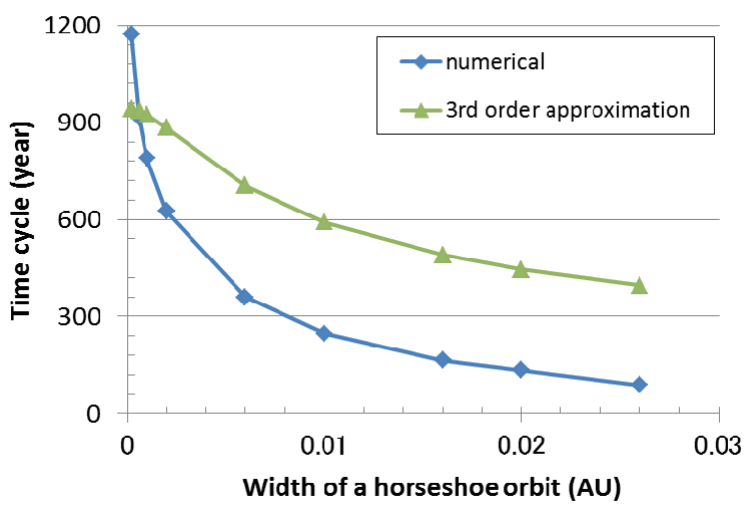

Fig. 7. Correlation between the time cycle and width of a horseshoe orbit in the case of analysis using variational method. 


\subsection{A horseshoe orbit subjected to SRP and P-R drag}

Substituting precondition Eq. (4.5) into Eq. (2.4) gives the following relationship about P-R drag.

$$
\begin{gathered}
f_{P \boldsymbol{R}}=-\frac{\beta \mu_{S}}{c r_{1}^{2}}\left\{\left(\boldsymbol{V} \cdot \frac{\boldsymbol{r}}{r}\right) \cdot \frac{\boldsymbol{r}}{r}+\boldsymbol{V}+\boldsymbol{\omega} \times \boldsymbol{r}\right\} \\
\because \boldsymbol{v}=\boldsymbol{V}+\boldsymbol{\omega} \times \boldsymbol{r}
\end{gathered}
$$

By substituting Eq. (4.22) and Eq. (4.27) into Eq. (4.1), the motion equation is expressed as,

$$
\begin{aligned}
& \ddot{r}-2 n r \dot{\theta}-n^{2} r=-\frac{\mu_{S}}{r^{2}}-\frac{\mu_{E}}{\left(2 r_{0}\right)^{2} \cos \frac{1}{2} \theta}+\frac{\beta \mu_{S}}{r^{2}}-\frac{\beta \mu_{S}}{c r^{2}} 2 \dot{r} \\
& r \ddot{\theta}+2 n \dot{r}=\frac{\mu_{E}}{\left(2 r_{0} \cos \frac{1}{2} \theta\right)^{2}} \sin \frac{1}{2} \theta-\frac{\beta \mu_{S}}{c r^{2}}(n \cdot r+r \dot{\theta})
\end{aligned}
$$

Eq. (4.29) can be linearized and normalized in the same way,

$$
\left[\begin{array}{c}
\ddot{r} \\
\ddot{\theta}
\end{array}\right]+\left(\begin{array}{cc}
\frac{2 \beta}{c^{\prime}} & -2 \\
2 & \frac{\beta}{\hat{c}}
\end{array}\right)\left[\begin{array}{c}
\dot{r} \\
\dot{\theta}
\end{array}\right]+\left(\begin{array}{cc}
-3+2 \beta & 0 \\
-\frac{\beta}{c^{\prime}} & -\frac{\mu_{1}}{8}
\end{array}\right)\left[\begin{array}{l}
r \\
\theta
\end{array}\right]=0
$$

where

$$
\begin{gathered}
\left(r^{\prime}-\Delta a\right) \rightarrow r,\left(\theta^{\prime}-\Delta \Theta\right) \rightarrow \theta \\
\Delta a=\frac{\beta-\frac{1}{4} \mu_{1}}{3+2 \beta}, \Delta \Theta=-\frac{\beta}{c^{\prime}} \frac{8}{\mu_{1}}\left(1-\frac{\beta-\frac{1}{4} \mu_{1}}{3+2 \beta}\right)>0
\end{gathered}
$$

The analytical solution of Eq. (4.30) is

$$
\begin{aligned}
& r=A_{l} e^{\alpha_{l}} \cos \left(\omega_{l} t+\gamma_{l}\right)+A_{s} e^{\alpha_{s}} \cos \left(\omega_{s} t+\gamma_{s}\right) \\
& \theta=B_{l} e^{\alpha_{l}} \sin \left(\omega_{l} t+\gamma_{l}\right)+B_{s} e^{\alpha_{s}} \sin \left(\omega_{s} t+\gamma_{s}\right)
\end{aligned}
$$

In the case of $\beta=0.1$, the coefficients in Eq. (4.33) is

$$
\begin{aligned}
& \alpha_{l}=2.00 \cdot 10^{-5}>0, \quad T_{l}=\frac{1}{\omega_{l}}=1069 \text { year } \\
& \alpha_{s}=-3.50 \cdot 10^{-5}<0, \quad T_{s}=\frac{1}{\omega_{s}}=0.913 \text { year }
\end{aligned}
$$

In the case of a horseshoe orbit subjected to SRP and P-R drag, the center of oscillation moves by $\Delta a$ and $\Delta \Theta$ respective. Fig. 9 shows a horseshoe orbit obtained from a numerical integration incorporating the effect of SRP and P-R drag. shape of a horseshoe orbit drastically changes by P-R drag.

A dust turns at an edge due to the Earth's gravity at $\phi=360^{\circ}$ and the other edge due to P-R drag at $\phi=80^{\circ}$ in Fig. 9. The orbit doesn't reach the leading end of the Earth and then doesn't encompass Lagrange point L3, L4. Therefore, it may be improper to describe the orbit as a horseshoe orbit. The velocity of a dust also becomes minimum at both edges. Therefore, If dust particles are trapped in a horseshoe orbit at the constant time interval, the number density of a dust particle would have peaks at $\phi=360^{\circ}$ and $\phi=80^{\circ}$

And the analytical solution Eq. (4.33) also indicates that the short period solution converges and the long period diverges with time, which means that the eccentricity of a dust decreases with time and the width and phase angle of a dust's orbit becomes larger and then a dust ends up departing from a horseshoe orbit.

Fig. 10 shows change of dust path with time and Fig. 12 shows time variation of semi-major axis. The result of numerical simulation revealed that once a dust becomes trapped in a horseshoe orbit, the width of the orbit becomes larger with time, and the dust ends up departing from the orbit as seen in Fig. 11. The time scale from becoming trapped to departing is about several thousand or tens of thousands year.

This behavior of a dust makes the prediction of a distribution more difficult, because the location of the edge of a horseshoe orbit continues to change with time. However, from numerical analysis, if we can obtain statistical information about Jacobi integral of dust particles trapped in a horseshoe orbit, it is possible to predict peaks of a number density of dust particles.

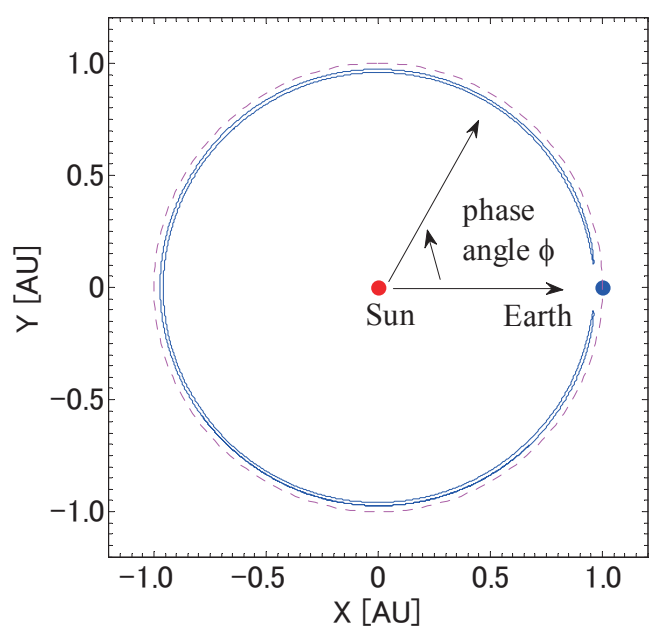

Fig. 8. Horseshoe orbit obtained from numerical integration of CR3BP incorporating SRP ( $\beta=0.1, \mathrm{~T}=180$ year). Dot-line is Earth's orbit and solid line is a horseshoe orbit. Under SRP, a horseshoe orbit is formed inside of the Earth's one.

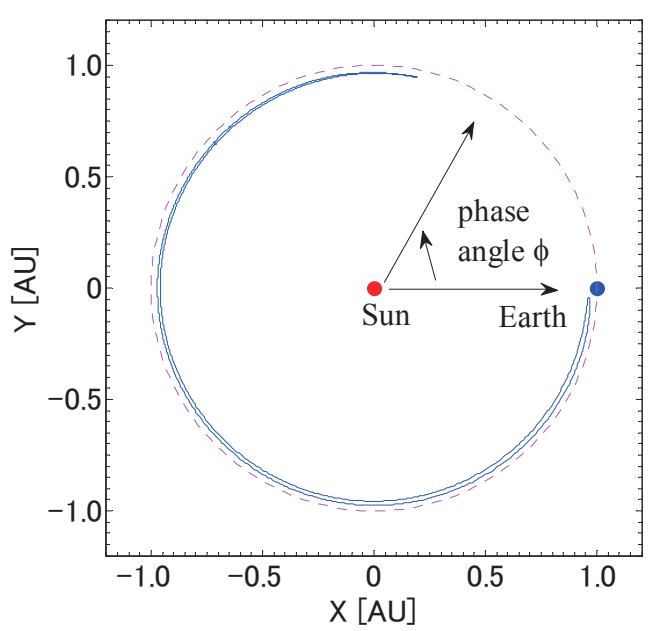

Fig. 9. Horseshoe orbit obtained from numerical integration of CR3BP incorporating the effect of SRP and P-R drag ( $\beta=0.1, \mathrm{~T}=187$ year). 

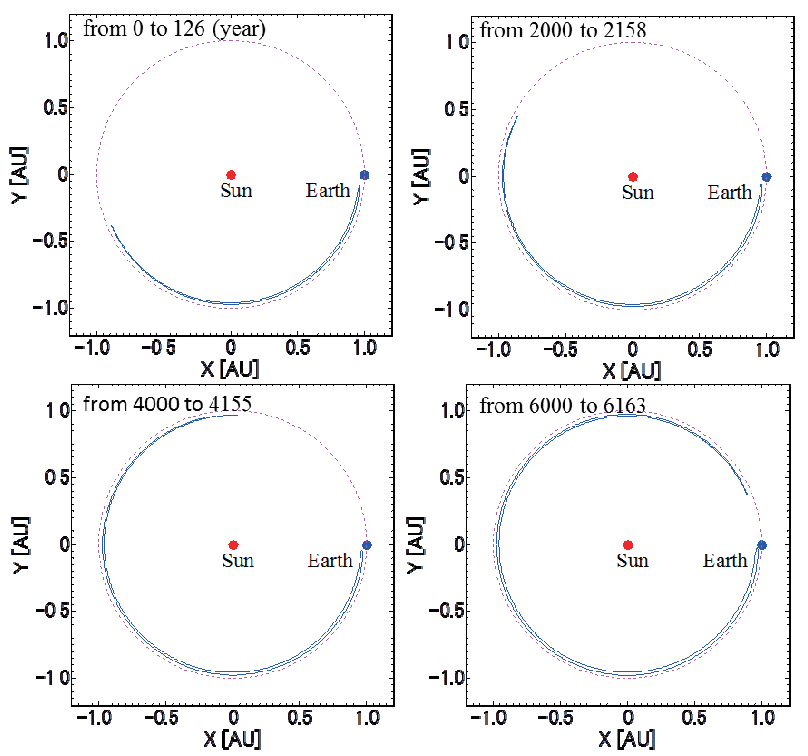

Fig. 10. Horseshoe orbits of a test particle at four different time periods. A dust became trapped in a horseshoe orbit at $\mathrm{T}=0$ (year), and departed from the orbit at about $\mathrm{T}=7000$ (year).
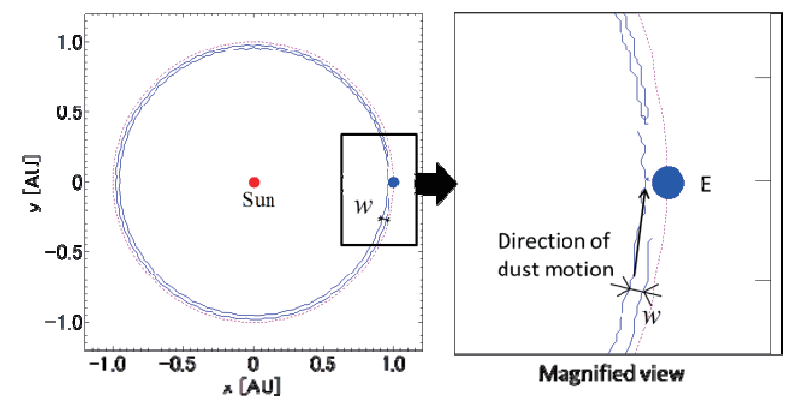

Fig. 11. An orbit of a dust particle which departed from horseshoe orbit. A dust particle on a horseshoe orbit whose width $w$ was large was not subjected to enough Earth's gravity to turn at the trailing side of the Earth, and then departed from a horseshoe orbit.

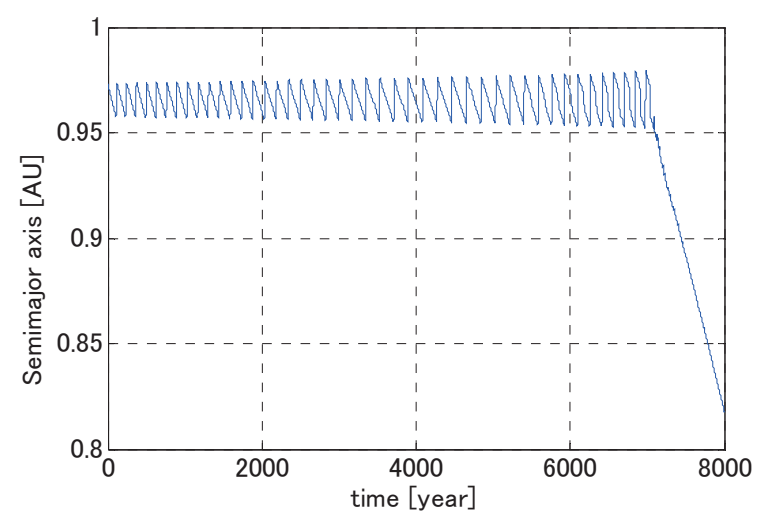

Fig. 12. Time variation of semi-major axis of the test particles in Fig. 10. The amplitude of the oscillation of semi-major axis became larger with time due to P-R drag, which indicates that the width of a horseshoe orbit became larger with time. After dust's departing from a horseshoe orbit, semi-major axis decreased rapidly and a dust spiraled into the Sun due to P-R drag.

\section{Conclusion}

This paper investigated the motion of a dust on a horseshoe orbit by analytical and numerical approaches.

The horseshoe orbit solution is obtained by coordinate transformations and linearization of external forces on the assumption that a horseshoe orbit is the oscillation centered at L3. The analytical solutions are consistent with results of numerical integration of non-linear equation of CR3BP.

This solution shows that the velocity of a dust becomes minimum at both ends of a horseshoe orbit which means that the number density of dust particles is maximum at both turned edges of a horseshoe orbit. And it is also found that in the case of a dust subjected to perturbation forces, a horseshoe orbit of a dust changes with time and end up departing from the orbit.

\section{References}

1) Motooka. N. : A Study on the Distribution of Dust Particles Trapped in Horseshoe Orbit, ISTS, 2009-b-24s

2) Donald. W. : The Restricted Three-Body Problem Including Radiation Pressure, The Astrophysical Journal, 1980, pp.337-342.

3) Klacka, J. : Poynting-Robertson Effect I. Equation of Motion, Earth, Moon and Planets 59, pp.41-59.

4) Klacka, J. : Poynting-Robertson Effect II. Perturbation equations, Earth, Moon and Planets 59, pp. 211-218. 